\title{
THE HOT-JUPITER KEPLER-17b: DISCOVERY, OBLIQUITY FROM STROBOSCOPIC STARSPOTS, AND ATMOSPHERIC CHARACTERIZATION
}

\author{
Jean-Michel Désert ${ }^{1}$, David Charbonneau ${ }^{1}$, Brice-Olivier Demory ${ }^{2}$, Sarah Ballard $^{1}$, Joshua A. Carter ${ }^{1}$, \\ Jonathan J. Fortney ${ }^{3}$, William D. Cochran ${ }^{4}$, Michael Endl ${ }^{4}$, Samuel N. Quinn ${ }^{1}$, Howard T. Isaacson ${ }^{5}$, \\ François Fressin $^{1}$, Lars A. Buchhave ${ }^{6}$, David W. Latham ${ }^{1}$, Heather A. Knutson ${ }^{5}$, Stephen T. Bryson ${ }^{7}$, \\ Guillermo Torres $^{1}$, Jason F. Rowe ${ }^{7}$, Natalie M. Batalha ${ }^{8}$, William J. Borucki ${ }^{7}$, Timothy M. Brown ${ }^{9}$, \\ Douglas A. Calddwell ${ }^{10}$, Jessie L. Christiansen ${ }^{7}$, Drake Deming ${ }^{11}$, Daniel C. Fabrycky ${ }^{3}$, Eric B. Ford ${ }^{12}$, \\ Ronald L. Gilliland ${ }^{13}$, Michaël Gillon ${ }^{14}$, Michaël R. HaAs ${ }^{7}$, Jon M. Jenkins ${ }^{10}$, Karen Kinemuchi ${ }^{15}$, David Koch ${ }^{7}$, \\ Jack J. Lissauer $^{1}$, Philip Lucas ${ }^{16}$, Fergal Mullally ${ }^{10}$, Phillip J. MacQueen ${ }^{4}$, Geoffrey W. Marcy ${ }^{5}$, \\ Dimitar D. Sasselov ${ }^{1}$, Sara Seager $^{2}$, Martin Still ${ }^{15}$, Peter Tenenbaum ${ }^{10}$, Kamal Uddin $^{17}$, and Joshua N. Winn ${ }^{2}$ \\ ${ }^{1}$ Harvard-Smithsonian Center for Astrophysics, 60 Garden Street, Cambridge, MA 02138, USA; jdesert@ cfa.harvard.edu \\ ${ }^{2}$ Massachusetts Institute of Technology, Cambridge, MA 02159, USA \\ ${ }^{3}$ Department of Astronomy and Astrophysics, University of California, Santa Cruz, CA 95064, USA \\ ${ }^{4}$ Department of Astronomy, University of Texas, Austin, USA \\ ${ }^{5}$ Department of Astronomy, University of California, Berkeley, CA 94720-3411, USA \\ ${ }^{6}$ Neils Bohr Institute, University of Copenhagen, DK-2100 Denmark \\ ${ }^{7}$ NASA Ames Research Center, Moffett Field, CA 94035, USA \\ ${ }^{8}$ San Jose State University, San Jose, CA 95192, USA \\ ${ }^{9}$ Las Cumbres Observatory Global Telescope, Goleta, CA 93117, USA \\ ${ }^{10}$ SETI Institute/NASA Ames Research Center, Moffett Field, CA 94035, USA \\ ${ }^{11}$ Solar System Exploration Division, NASA Goddard Space Flight Center, Greenbelt, MD 20771, USA \\ ${ }^{12}$ Department of Astronomy, University of Florida, Gainesville, FL 32611, USA \\ ${ }^{13}$ Space Telescope Science Institute, Baltimore, MD 21218, USA \\ ${ }^{14}$ Institut d'Astrophysique et de Géophysique, Université de Liège, Allée du 6 Août 17, Bat. B5C, 4000 Liège, Belgium \\ ${ }^{15}$ Bay Area Environmental Research Inst./NASA Ames Research Center, Moffett Field, CA 94035, USA \\ ${ }^{16}$ Centre for Astrophyiscs Research, University of Hertfordshire, College Lane, Hatfield, AL10 9AB, UK \\ ${ }^{17}$ Orbital Sciences Corporation/NASA Ames Research Center, Moffett Field, CA 94035, USA \\ Received 2011 July 25; accepted 2011 October 14; published 2011 November 4
}

\begin{abstract}
This paper reports the discovery and characterization of the transiting hot giant exoplanet Kepler-17b. The planet has an orbital period of 1.486 days, and radial velocity measurements from the Hobby-Eberly Telescope show a Doppler signal of $419.5_{-15.6}^{+13.3} \mathrm{~m} \mathrm{~s}^{-1}$. From a transit-based estimate of the host star's mean density, combined with an estimate of the stellar effective temperature $T_{\text {eff }}=5630 \pm 100$ from high-resolution spectra, we infer a stellar host mass of $1.06 \pm 0.07 M_{\odot}$ and a stellar radius of $1.02 \pm 0.03 R_{\odot}$. We estimate the planet mass and radius to be $M_{\mathrm{P}}=2.45 \pm 0.11 M_{\mathrm{J}}$ and $R_{\mathrm{P}}=1.31 \pm 0.02 R_{\mathrm{J}}$. The host star is active, with dark spots that are frequently occulted by the planet. The continuous monitoring of the star reveals a stellar rotation period of 11.89 days, eight times the planet's orbital period; this period ratio produces stroboscopic effects on the occulted starspots. The temporal pattern of these spot-crossing events shows that the planet's orbit is prograde and the star's obliquity is smaller than $15^{\circ}$. We detected planetary occultations of Kepler-17b with both the Kepler and Spitzer Space Telescopes. We use these observations to constrain the eccentricity, $e$, and find that it is consistent with a circular orbit $(e<0.011)$. The brightness temperatures of the planet's infrared bandpasses are $T_{3.6 \mu \mathrm{m}}=1880 \pm 100 \mathrm{~K}$ and $T_{4.5} \mu \mathrm{m}=1770 \pm 150 \mathrm{~K}$. We measure the optical geometric albedo $A_{g}$ in the Kepler bandpass and find $A_{g}=0.10 \pm 0.02$. The observations are best described by atmospheric models for which most of the incident energy is re-radiated away from the day side.
\end{abstract}

Key words: eclipses - planetary systems - stars: individual (Kepler-17b, KIC 10619192 , 2MASS 19533486+4748540) - techniques: photometric

Online-only material: color figures

\section{INTRODUCTION}

NASA's Kepler mission is a space-based photometric telescope dedicated to finding Earth-size planets in the habitable zones of their host stars and to determining the frequency and characteristics of planetary systems around Sun-like stars (Borucki et al. 2010). While monitoring nearly continuously the brightness of about 150,000 dwarf stars since 2009 May to achieve the above goals, it accumulates an extraordinary volume of data allowing atmospheric studies of the transiting hotJupiters present in the field of view (Borucki et al. 2009). The first four months of observations were released and 1235 transiting planet candidates were reported (Borucki et al. 2011). Because of their short periods and relatively deep transit depths, several hot-Jupiter candidates were discovered early on during the first weeks of the mission. Among those, the Kepler Object of Interest, KOI-203, was identified as a promising target, was selected for follow-up studies, and placed on the short-cadence (SC) target list for subsequent quarters. This is the object of interest of the current study.

The highly irradiated transiting hot-Jupiters currently provide the best opportunities for studying exoplanetary atmospheres in 
emission, during planetary occultations, when the exoplanets pass behind their parent stars (Seager et al. 2000; Sudarsky et al. 2000; Fortney et al. 2005; Barman et al. 2005). Light emitted from exoplanets was first detected from space at infrared wavelengths (Charbonneau et al. 2005; Deming et al. 2005) and more recently in the optical (Alonso et al. 2009; Snellen et al. 2009; Borucki et al. 2009; Alonso et al. 2010) using the CoRoT and Kepler Space Telescopes. Notably, Rowe et al. (2006, 2008) used the Microvariablity and Oscillations of Stars (MOST) telescope to place a very stringent upper limit on the depth of the occultation of HD 209458b.

The hot-Jupiters detected by CoRoT and Kepler are particularly good targets for studying planetary atmospheres, because the precise and nearly uninterrupted photometric surveillance provided by the space satellites can allow the planetary occultations to be detected at optical wavelengths (Snellen et al. 2009; Borucki et al. 2009; Désert et al. 2011b). Obtaining multiple wavelength observations of the relative depths of planetary occultations is necessary to constrain the broad band emergent spectra. Such observations are fundamental to understanding the energy budget of these objects (Sudarsky et al. 2003; Burrows et al. 2005, 2007, 2008; Spiegel \& Burrows 2010) and for comparative exoplanetology.

We confirm here the planetary status of KOI-203, designating it as Kepler-17b, and study its atmosphere. We first describe the Kepler observations and transit modeling as well as the follow-up observations used to confirm this planet, including an orbital solution using radial velocities obtained with the High Resolution Spectrometer (HRS) at the Hobby-Eberly Telescope (HET). We then study the impact of the stellar variability on the transit light curves and we use occulted dark starspots to constrain the stellar obliquity. We finally combine occultation measurements obtained in the optical with Kepler and in the infrared with Spitzer to learn about the atmospheric properties of Kepler-17b.

We first describe the observations, time series, and analysis of the Kepler photometry in Section 2, then we describe the followup observations that confirm the planet detection in Section 3 and then discuss the stellar properties in Section 4. In Section 5, we describe the occultation measurements obtained from the visible and infrared. We present a global Monte Carlo analysis of the complete sample of observations in Section 6 and finally discuss our findings in Section 7.

\section{Kepler OBSERVATIONS}

Observations of the Kepler field commenced in 2009 May with Quarter $0(\mathrm{Q} 0)$; the data that we describe here are the Kepler science data of Kepler-17 from Quarter 0 to 6 (Q0-Q6). The Kepler observations were gathered almost continuously during 16.7 months. These observations have been reduced and detrended by the Kepler pipeline (Jenkins et al. 2010b). The Kepler bandpass spans 423-897 $\mathrm{nm}$ for which the response is greater than 5\% (Batalha et al. 2010; Bryson et al. 2010). This wavelength domain is roughly equivalent to the $V+R$ band (Koch et al. 2010a). The target Kepler-17 was identified in the Kepler Input Catalog (Brown et al. 2011; 2MASS $19533486+4748540$, KIC 10619192, $r=14.08 \mathrm{mag}$ ). Because of the transit-like events, the object was then considered for follow-up studies, and identified as Kepler Object of Interest KOI-203. The pipeline produces both calibrated light curves (P.A. data) for individual analysis and corrected light curves (pre-search data conditioning) which are used to search for transits. This paper presents results which are measured from
P.A. data only. They consist of long cadence integration time (29.426 minutes) for Quarters 0 and 1 (Caldwell et al. 2010; Jenkins et al. 2010a) and long and short cadence (1 minute) for Quarters 2-6 (Gilliland et al. 2010). The pipeline provides time series with times in barycentric-corrected Julian days and flux in photoelectrons per cadence. The raw nearly continuous photometry of Kepler-17 is presented in Figure 1. We measure the transit parameters from the Kepler observations as described below (see Section 6). We present the normalized, phase-folded, and combined transit light curve obtained at SC in Figure 2 from which we measure the transit parameters.

\section{FOLLOW-UP OBSERVATIONS}

\subsection{Reconnaissance Spectroscopy}

As described in detail by Gautier et al. (2010), the follow-up observations of Kepler planet candidates involve reconnaissance spectroscopy to look for evidence of astrophysical false positives responsible for the observed transits. These false positives include single- and double-lined binaries, certain types of hierarchical triples, and even some background eclipsing binaries, which would show velocity variations and/or composite spectra that are readily detectable by the modest facilities used for these reconnaissance observations. As described below, we also use these spectra to estimate the effective temperature, surface gravity, metallicity, and rotational and radial velocities of the host star.

On 2011 April 25 UT, we obtained a spectrum of Kepler-17 using the fiber-fed Tillinghast Reflector Echelle Spectrograph (TRES; Fürész 2008) on the $1.5 \mathrm{~m}$ Tillinghast Reflector at the Fred Lawrence Whipple Observatory, on Mt. Hopkins, AZ. The spectrum was taken with the medium fiber, which has a resolving power of $\lambda / \Delta \lambda=44,000$ and a wavelength coverage of about 3850-9100 $\AA$. The exposure time was 80 minutes. The spectrum was extracted and rectified to intensity versus wavelength using standard procedures described by Buchhave et al. (2010). The extracted spectrum has a signal-to-noise ratio $(\mathrm{S} / \mathrm{N})$ of 17.5 per resolution element.

We performed cross-correlations against a grid of synthetic stellar spectra, calculated by John Laird for a grid of Kurucz model atmospheres (Kurucz 1979), using a line list developed by Jon Morse. The grid is coarse- $250 \mathrm{~K}$ in effective temperature, $T_{\text {eff }} ; 0.5$ in $\log$ surface gravity, $\log g ; 0.5$ in $\log$ of the metallicity compared to the sun, $[\mathrm{M} / \mathrm{H}]$; and $2 \mathrm{~km} \mathrm{~s}^{-1}$ in rotational velocity, $V_{\text {rot }}$-so rather than simply adopting the parameters from the template with the best correlation coefficient, we fit a surface to the correlation peak heights to arrive at a refined classification. However, given the degeneracies between $T_{\text {eff }}, \log g$, and $[\mathrm{M} / \mathrm{H}]$, the quality of the spectrum is not sufficient to determine all three at once. As such, we have fixed $\log g=4.42 \pm 0.015$ as inferred from the best fit of the Kepler photometry. The analysis yields $T_{\text {eff }}=5630 \pm 100 \mathrm{~K},[\mathrm{M} / \mathrm{H}]=0.3 \pm 0.1$, $v \sin i=4.7 \pm 1.0 \mathrm{~km} \mathrm{~s}^{-1}$. When corrected for the orbital motion of Kepler-17 and the TRES zero-point offset, determined by long-term monitoring of the IAU RV standard HD 182488 , we find the absolute mean systemic velocity of Kepler-17 to be $-23.82 \pm 0.10 \mathrm{~km} \mathrm{~s}^{-1}$. Note that this does not include any uncertainty in the absolute velocity of HD 182488, which we take to be $-21.508 \mathrm{~km} \mathrm{~s}^{-1}$, as observed by Nidever et al. (2002).

We also obtained Keck HIRES spectra and estimated the line strengths of $S_{\mathrm{HK}}=0.322 \pm 0.01$ and $\log R_{\mathrm{HK}}^{\prime}=-4.61$ for Kepler-17 (assuming $B-V=0.82$ ). The Ca II $\mathrm{H} \& \mathrm{~K}$ line 


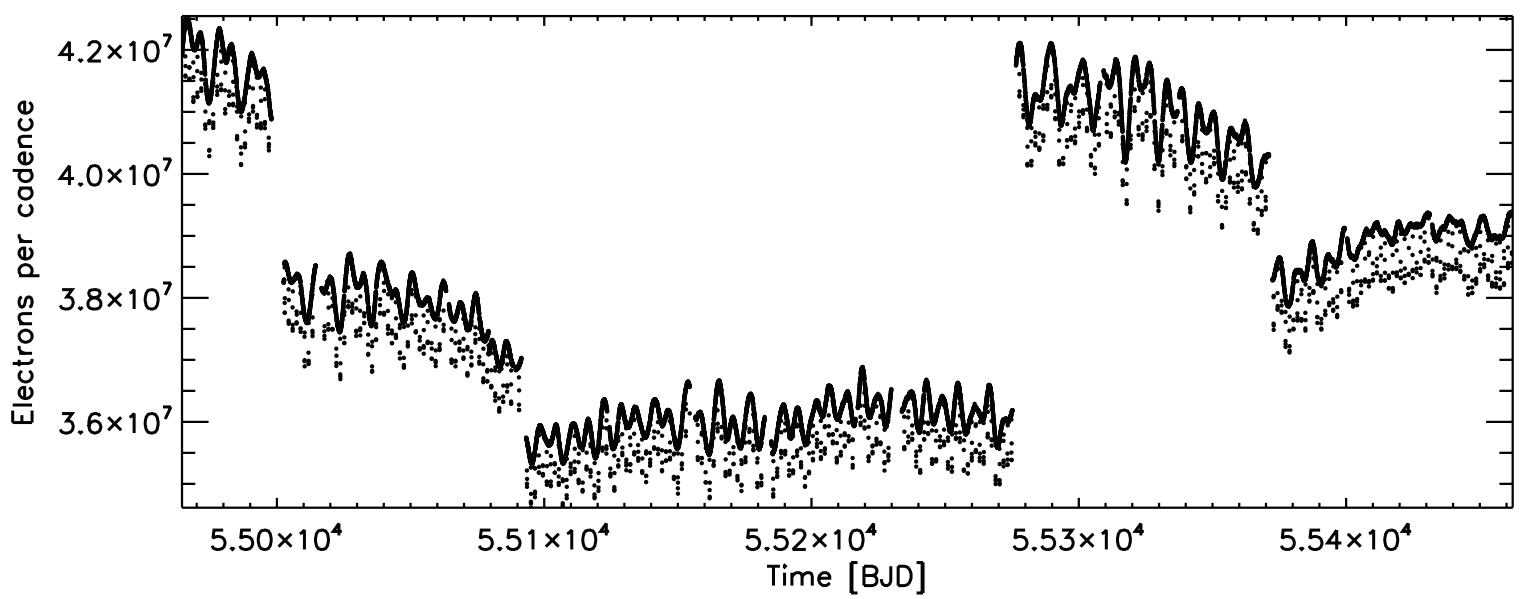

Figure 1. Kepler raw long-cadence light curve of Kepler-17, from Quarter 0 to Quarter 6. The drop of flux occurring every 1.48 days corresponds to the planetary transits. The stellar activity shows $3 \%$ flux variation and with a period of 11.89 days.
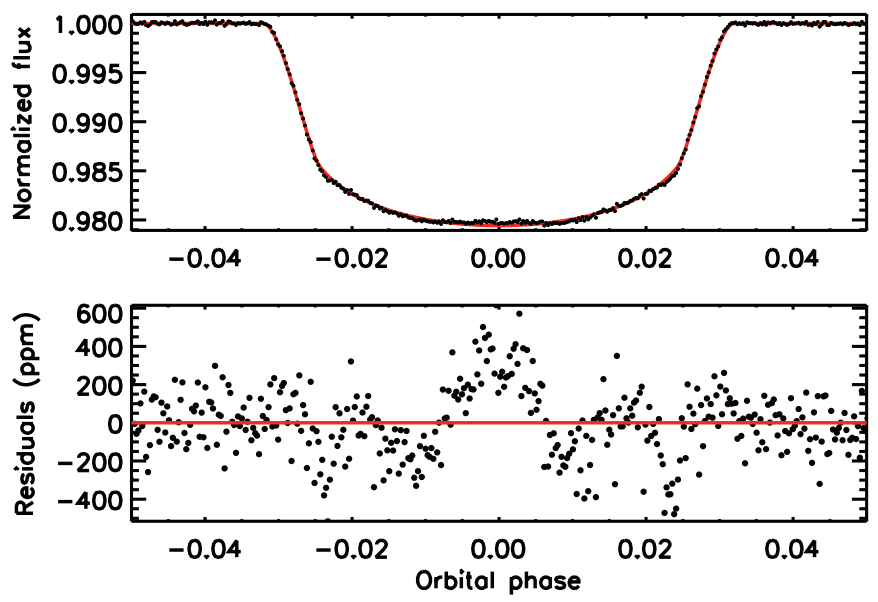

Figure 2. Top panel: Kepler transit light curve of Kepler-17b phase-folded, normalized and binned by $30 \mathrm{~s}$. The best-fit model of this light curve is overplotted in red. The residuals from the best-fit transit light curve are plotted in the bottom panel. Occulted stellar spots produce a symmetrical pattern in phase in the residuals (see Section 7.2.1).

(A color version of this figure is available in the online journal.)

strengths are a good indicator of the stellar activity (Isaacson \& Fischer 2010).

\subsection{Imaging}

The astrometry derived from the Kepler images themselves, when combined with high-resolution images of the target neighborhood, provides a very powerful tool for identifying background eclipsing binaries blended with and contaminating the target images (Batalha et al. 2010). The astrometry of Kepler-17 indicated no significant offset during transits in any quarter, and computed offsets are well within the formal $3 \sigma$ radius of confusion. Therefore, Kepler-17 is considered to be the source for the transits observed in the Kepler light curves.

We obtained an I-band image at the Lick Observatory $1 \mathrm{~m}$, Nickel telescope with the Direct Imaging Camera (see Figure 3). The 1".0 seeing revealed no companions from $2^{\prime \prime}$ to $5^{\prime \prime}$ from the star's center, down to a limit of 19th magnitude. Similar conclusions were reached using United Kingdom Infrared Telescope (UKIRT) $J$-band images (Figure 3).
Table 1

Relative Radial-velocity Measurements of Kepler-17

\begin{tabular}{lcrrrr}
\hline \hline $\begin{array}{l}\text { HJD } \\
\text { (days) }\end{array}$ & $\begin{array}{c}\text { Phase } \\
\text { (cycles) }\end{array}$ & $\begin{array}{c}\text { RV } \\
\left(\mathrm{m} \mathrm{s}^{-1}\right)\end{array}$ & $\begin{array}{c} \pm \sigma_{\mathrm{RV}} \\
\left(\mathrm{m} \mathrm{s}^{-1}\right)\end{array}$ & $\begin{array}{c}\mathrm{BS} \\
\left(\mathrm{m} \mathrm{s}^{-1}\right)\end{array}$ & $\begin{array}{r} \pm \sigma_{\mathrm{BS}} \\
\left(\mathrm{m} \mathrm{s}^{-1}\right)\end{array}$ \\
\hline 2455430.839929 & 313.013 & -142.61 & 67.08 & -77.7 & 114.6 \\
2455431.812259 & 313.667 & 325.71 & 49.17 & -23.8 & 72.4 \\
2455432.819992 & 314.345 & -300.32 & 90.74 & 42.2 & 68.9 \\
2455442.794995 & 321.060 & -119.08 & 43.22 & 45.6 & 68.2 \\
2455443.765027 & 325.080 & 528.51 & 198.75 & 483.3 & 276.7 \\
2455448.768015 & 327.760 & -158.75 & 51.43 & -110.9 & 218.2 \\
2455452.748541 & 341.195 & 358.36 & 61.44 & -50.3 & 67.7 \\
2455472.707059 & 346.565 & -413.96 & 25.96 & -113.8 & 80.9 \\
2455480.686032 & 355.957 & 138.23 & 56.24 & -116.1 & 70.4 \\
2455494.639064 & 357.302 & 83.68 & 36.83 & -47.9 & 75.7 \\
2455496.636013 & 372.077 & -511.48 & 60.28 & -36.6 & 75.3 \\
2455518.588037 & 372.747 & -205.45 & 56.70 & 1.2 & 67.6 \\
2455522.579531 & 374.764 & 417.16 & 28.10 & -121.9 & 34.2 \\
\hline
\end{tabular}

\subsection{Radial Velocity}

We obtained precise radial velocity (RV) follow-up observations of Kepler-17 with the HET (Ramsey et al. 1998) and its HRS spectrograph (Tull 1998) at McDonald Observatory. Kepler-17 was observed ten times in the 2010 observing season, from 2010 August 22 until 2010 November 22. The instrumental setup and observing mode are described in more detail in Endl et al. (2011). Kepler-17b is the second planet confirmed with HET after Kepler-15b (Endl et al. 2011). We employed a "snap shot" strategy, using relatively short exposures of $1200 \mathrm{~s}$, that yield an $\mathrm{S} / \mathrm{N}$ sufficient to detect the radial velocity signal of a hot-Jupiter. Thirteen spectra were taken with the $I_{2}$-cell in the light path to compute precise differential RVs. These spectra have a typical $\mathrm{S} / \mathrm{N}$ of 32 per resolution element. The radial velocity data are listed in Table 1.

We use Gaussfit, the generalized least-squares software of Jefferys et al. (1988) to fit a Keplerian orbit to the HRS radial velocity data. Only the velocity zero point and the radial velocity semi-amplitude $K$ are included as free parameters in the fitting process. We first fitted the radial velocity data alone, requiring the orbit to be circular $(e=0)$ and adopting the ephemeris derived from the Kepler photometry. The best-fit orbit has a $K$ of $420 \pm 15 \mathrm{~m} \mathrm{~s}^{-1}$, a $\chi_{\text {red }}^{2}$ of 0.9 , and a residual rms scatter around the fit of $52 \mathrm{~m} \mathrm{~s}^{-1}$. The radial velocity data and the orbital solution are shown in Figure 4. 

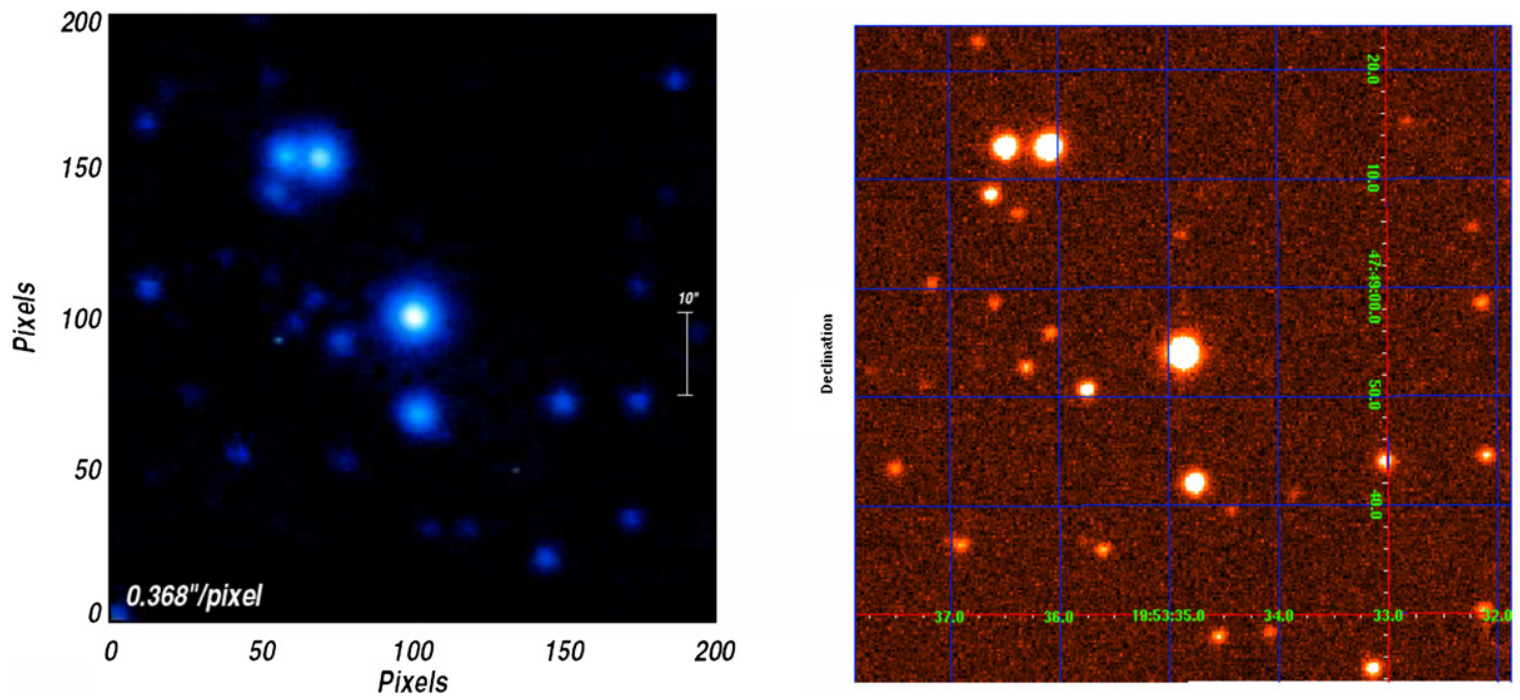

Figure 3. Left panel shows an $I$-band natural seeing image of Kepler-17 taken with the Lick 1-meter telescope $\left(1^{\prime} \cdot 2 \times 1\right.$ ' 2$)$. The right panel shows a $J$-band image of Kepler-17 taken with the wide field camera (WFCAM, $1^{\prime} \times 1^{\prime}$ ) on the United Kingdom Infrared Telescope (UKIRT). These images confirm that there are no companions from $2^{\prime \prime}$ to $5^{\prime \prime}$ from the star's center, down to a limit of 19th magnitude.

(A color version of this figure is available in the online journal.)

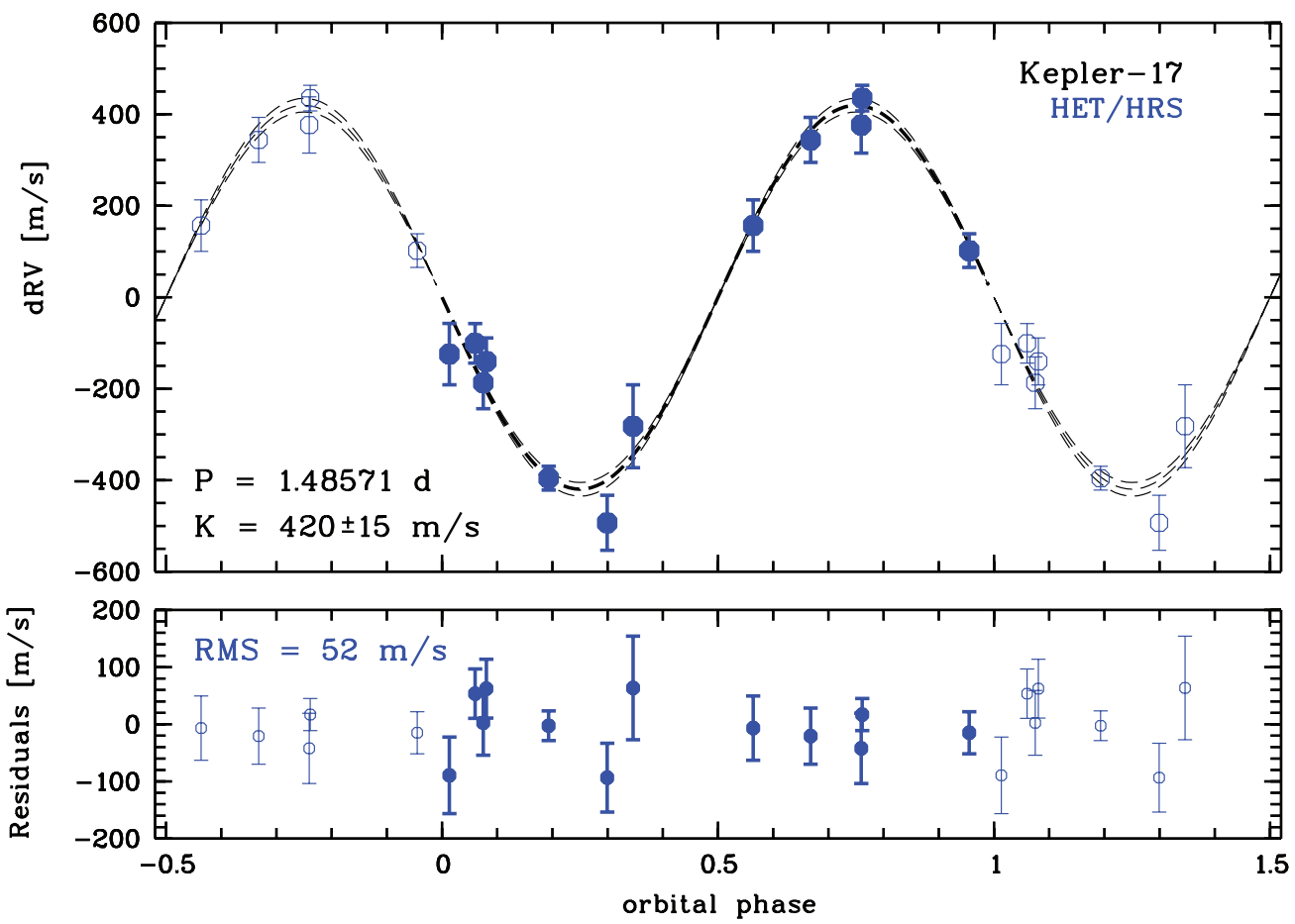

Figure 4. Orbital solution for Kepler-17. Top panel: the observed radial velocities obtained with HET HRS are plotted together with the velocity curve for a circular orbit with the period and time of transit fixed by the photometric ephemeris. The thick dashed line corresponds to the best fit and the thin dashed lines correspond to models with $\pm 1 \sigma$ error on the parameter K. The $\gamma$ velocity has been subtracted from the relative velocities here and in Table 1, and thus the center-of-mass velocity for the orbital solution is 0 by definition. Bottom panel: the velocity residuals from the orbital solution. The rms of the velocity residuals is $52 \mathrm{~m} \mathrm{~s}^{-1}$.

(A color version of this figure is available in the online journal.)

We determined the spectral line bisectors, which are a measure of line asymmetry, from the HET spectra to test if the radial velocity variations could be caused by distortions in the spectral line profiles due to contamination from a nearby unresolved eclipsing binary. We can only use a small fraction of the available spectral range that is not contaminated by the iodine absorption cell (5000-6400 ̊) and thus the uncertainties in the bisector velocity span (BVS) are quite large with an average uncertainty of $99 \mathrm{~m} \mathrm{~s}^{-1}$. The rms of the bisector measurements is $146 \mathrm{~m} \mathrm{~s}^{-1}$. There is no evidence of a correlation between the velocities and the bisectors, which supports the interpretation that the velocity variations are due to a planetary companion (e.g., Queloz et al. 2001).

\section{STELLAR PARAMETERS}

We derive the mass, radius, and age of the host star using the method described by Torres et al. (2008). We first created a set of stellar evolution models from the Yonsei-Yale $\left(\mathrm{Y}^{2}\right)$ series by $\mathrm{Yi}$ et al. (2001), with corrections from Demarque et al. (2004). We 
employed their interpolation software which accepts as inputs the age of the star, the iron abundance, and the abundance of $\alpha$-elements (relative to solar) for which we assume the solar value, and outputs a grid of stellar isochrones corresponding to a range of masses. We evaluated a set of isochrones at ages from 0.1 to $14 \mathrm{Gyr}$ (at intervals of $0.1 \mathrm{Gyr}$ ) and stellar metallicities spanning a range of $3 \sigma$ (at intervals of $0.01 \mathrm{dex}$ ) from the best-fit metallicity derived from spectra of $[\mathrm{Fe} / \mathrm{H}]=$ $0.3 \pm 0.1$. We then performed a spline interpolation of each output table at a resolution of 0.005 in effective temperature $T_{\text {eff }}$, the $\log$ of the surface gravity $\log (g)$, and the stellar luminosity $L_{\star}$. We evaluated the physical radius corresponding to each stellar model via $\log (g)$ and the mass of the star, though it is also possible to convert to physical radius using the model stellar luminosity and effective temperature (assuming $L_{\star}=4 \pi R_{\star}^{2} \sigma T^{4}$ ); in practice these conversions give identical results.

We fitted for the stellar mass and radius using Newton's version of Kepler's third law in the manner employed by Seager \& Mallén-Ornelas (2003), Sozzetti et al. (2007), and Torres et al. (2008). We assumed that the planetary mass is negligible when compared to the mass of the host star. Using the Markov Chain Monte Carlo (MCMC) sequence of $a / R_{\star}$ and generating a series of Gaussian random realizations of $[\mathrm{Fe} / \mathrm{H}]$ and $T_{\text {eff }}$ using the values and error bars derived from spectroscopy of $T_{\text {eff }}=$ $5630 \pm 100 \mathrm{~K}$ and $[\mathrm{Fe} / \mathrm{H}]=0.3 \pm 0.1$, respectively, we located the best isochrone fit at each realization using the $\chi^{2}$ goodness of fit given in Equation (1):

$$
\chi^{2}=\left(\frac{\Delta a / R_{\star}}{\sigma_{a / R_{\star}}}\right)^{2}+\left(\frac{\Delta T_{\text {eff }}}{\sigma_{T_{\text {eff }}}}\right)^{2}+\left(\frac{\Delta[\mathrm{Fe} / \mathrm{H}]}{\sigma_{[\mathrm{Fe} / \mathrm{H}]}}\right)^{2} .
$$

Using the output of the MCMC chain of $a / R_{\star}$ ensures that any correlations between parameters, which are reflected in the chain, are properly incorporated into our estimate of the stellar parameters.

We then assign a weight to the likelihood of each stellar model in the chain, applying a prior for the initial mass function (IMF) which assumes a Salpeter index. The number of stars of each mass and age, per 1000 stars, is generated by the interpolation software provided by Yi et al. (2001) for several IMF assumptions, including the Salpeter IMF. We designate the weight assigned to each stellar model in the chain by normalizing to the highest IMF value within the sample; in practice, the weights vary from 0.3 to 1 (from the least to most likely). The IMF prior changes the final answer by less than half a $\sigma$ for all parameters. We then incorporate this likelihood by discarding members of the chain according to their weight, where the weight is equal to the likelihood of remaining sampling; about $40 \%$ of the original chain remains intact after this stage. The value for each stellar parameter is then assigned from the median of this weighted distribution, with the error bars assigned from the nearest $\pm 34 \%$ of values. In this way, we find $M_{\star}=1.061_{-0.040}^{+0.045} M_{\odot}, R_{\star}=1.019 \pm 0.014 R_{\odot}$, and an age $=2.9_{-1.6}^{+1.5}$ Gyr. These uncertainties are statistical as they exclude possible systematic uncertainties in the stellar models. The best-fit solution is presented in Figure 5.

We caution that stellar isochrones are only poorly constrained for this faint star. The estimated error bars on the stellar parameters are smaller than what is expected for such a star of this magnitude. This is because we set the stellar gravity as a fixed value in our analysis, since the stellar spectrum has a low $\mathrm{S} / \mathrm{N}$. We set the stellar gravity to the value we

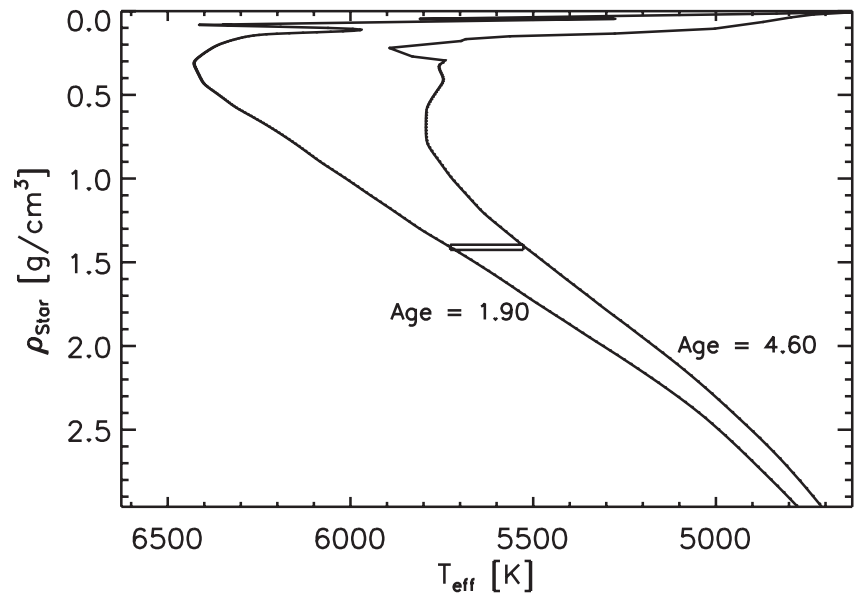

Figure 5. Stellar isochrones in the range of the observed $T_{\text {eff }}$ and $\rho_{\star}$. The small rectangle corresponds to the range of possible solutions.

derive from the stellar density measured using the Kepler photometry (see Section 3.1). The quality of the combined transit light curve allows us to measure the stellar density with a high precision that tightly constrains the isochrone fits (see Figure 5). However, a recent study of the physical properties of the stellar components of the transiting exoplanets by Southworth (2011) shows that the current stellar models are not determined at better than $1 \%$ in terms of radius and $2 \%$ in mass (median values); more conservative values are $3 \%$ and $5 \%$ for the stellar radius and mass, respectively. We adopt these conservative values and propagate the systematic errors arising from the dependence on stellar theory to the final errors. Adding the statistical and systematical error quadratically, we find $M_{\star}=1.061 \pm 0.07 M_{\odot}, R_{\star}=1.019 \pm 0.03 R_{\odot}$. This study shows that Kepler-17 is consistent with a main-sequence $G$ dwarf star, its radius and $T_{\text {eff }}$ are indistinguishable from solar.

\section{OCCULTATIONS OF Kepler-17b}

\subsection{Occultations from the Kepler Photometry}

The first method we use to measure the occultations from the Kepler observations is described by Désert et al. (2011b). We search for the occultation events in the SC light curves. We normalize and combine 173 occultations events. The best fit and the maximum depth as a function of the orbital phase are found very close to the orbital phase of 0.5 as expected for a circular orbit. We estimate the significance of this detection by measuring the occultation depth, ephemeris, and associated errors using a bootstrap Monte Carlo analysis. We find that the planet has an occultation depth of $58 \pm 10 \mathrm{ppm}$.

In a second method, we include the occultations in a MCMC global analysis as described below in Section 6. We present the normalized, folded, combined, and binned per 15 minute light curve obtained at long cadence in Figure 6 from which we measure the occultation depth with the MCMC.

\subsection{Spitzer Observations and Photometry}

The method we use to measure the occultations from the Spitzer observations is described in Désert et al. (2009). Kepler-17 was observed during four occultations between 2010 August and 2011 January with Warm-Spitzer/IRAC (Werner et al. 2004; Fazio et al. 2004) at 3.6 and $4.5 \mu \mathrm{m}$ (program ID 60028). Two occultations were gathered per bandpass and 

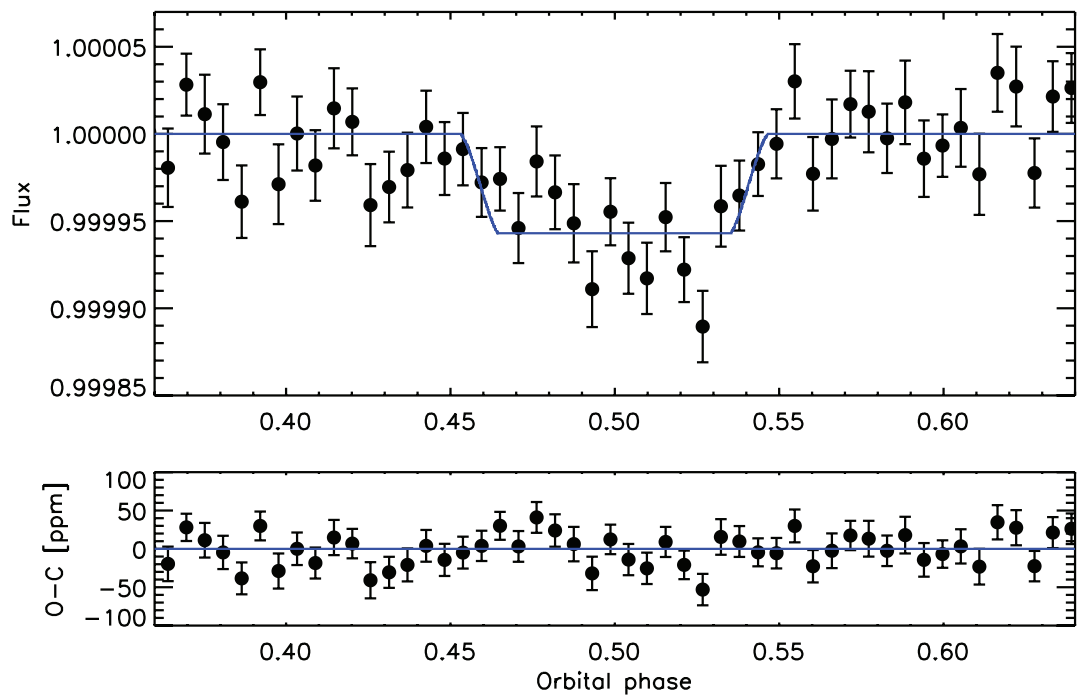

Figure 6. Phase-folded and normalized Kepler occultation light curve of Kepler-17b binned by 15 minutes (top panel). The best-fit model of the occultation in the Kepler bandpass is overplotted in blue and the residual from this best fit are shown in the bottom panel.

(A color version of this figure is available in the online journal.)

Table 2

Warm-Spitzer Observations

\begin{tabular}{lccccccc}
\hline \hline Visit & AOR & $\begin{array}{c}\text { Wavelength } \\
(\mu \mathrm{m})\end{array}$ & $\begin{array}{c}\text { Obs. Date } \\
(\mathrm{UT})\end{array}$ & Select. Points & $\begin{array}{c}\text { Depth } \\
(\%)\end{array}$ & Weighted. Avg. Depth & $\begin{array}{c}\text { Bright. } T \\
(\mathrm{~K})\end{array}$ \\
\hline 1 & 40252416 & 3.6 & 2010 Aug 29 & 2266 & $0.311_{-0.060}^{+0.045}$ & $\ldots$ & \\
3 & 40251904 & 3.6 & 2010 Sep 16 & 2301 & $0.235_{-0.030}^{+0.030}$ & $0.250 \% \pm 0.030 \%$ & $1880 \pm 110$ \\
2 & 40252160 & 4.5 & 2010 Aug 30 & 2315 & $0.270_{-0.060}^{+0.040}$ & $\ldots$ & \\
4 & 40251648 & 4.5 & 2010 Sep 18 & 2313 & $0.346_{-0.050}^{+0.060}$ & $0.310 \% \pm 0.035 \%$ & $1770 \pm 150$
\end{tabular}

each visit lasted approximately $8.6 \mathrm{hr}$. The data were obtained in full-frame mode $(256 \times 256$ pixels $)$ with an exposure time of $30.0 \mathrm{~s}$ per image and yielded 2461 images per visit. The method we use to produce photometric time series from the images is described in Désert et al. (2011a). It consists of finding the centroid position of the stellar point-spread function (PSF) and performing aperture photometry using a circular aperture on individual Basic Calibrated Data (BCD) images delivered by the Spitzer archive. The optimal apertures were found to be 2.5 pixels in radius. The final photometric measurements used are presented in Table 2. The raw time series are presented in the top panels of Figure 7. Telescope pointing drift results in fluctuations of the stellar centroid position, which, in combination with intra-pixel sensitivity variations, produces systematic noise in the raw light curves (upper panel, Figure 7). A description of this effect, known as the pixel-phase effect, is given in the Spitzer/IRAC data handbook (Reach et al. 2006) and is well known in exoplanetary studies (e.g., Charbonneau et al. 2005; Knutson et al. 2008). To correct the light curve, we define a baseline function that is the sum of a linear function of time and a quadratic function (with four parameters) of the $X$ and $Y$ centroid positions. We find that the point-to-point scatter in the photometry gives a typical $\mathrm{S} / \mathrm{N}$ of 90 and 110 per image at 3.6 and $4.5 \mu \mathrm{m}$, respectively. These correspond to $85 \%$ of the theoretical $\mathrm{S} / \mathrm{N}$.

We simultaneously fit the instrumental functions with all the parameters, measured the occultation depths for each individual visit, and report the values in Table 2 . The measurements per bandpass agree at the $1 \sigma$ level. The weighted mean of the transit depths are $0.250 \% \pm 0.030 \%$ and $0.310 \% \pm 0.035 \%$ at 3.6 and $4.5 \mu \mathrm{m}$, respectively.
We also included the Spitzer observations in the global MCMC analysis described below.

\section{MARKOV CHAIN MONTE CARLO ANALYSIS}

The analysis of the Kepler photometry and the determination of the stellar and planetary parameters for Kepler-17 follows procedures similar to those reported in Borucki et al. (2010). We check and confirm these results by an independent analysis with the global MCMC algorithm presented in Gillon et al. (2009, 2010). This MCMC implementation uses the Metropolis-Hasting algorithm to perform the sampling. Input data to the MCMC include the Kepler and Spitzer photometric measurements and the radial velocity data.

Kepler SC data allow a precise determination of the transit parameters and more specifically allow a fit for the limbdarkening (LD) coefficients. We therefore assumed a quadratic law and used $c_{1}=2 u_{1}+u_{2}$ and $c_{2}=u_{1}-2 u_{2}$ as jump parameters, where $u_{1}$ and $u_{2}$ are the quadratic coefficients. Those linear combinations help in minimizing correlations on the uncertainties of $u_{1}$ and $u_{2}$ (Holman et al. 2006).

The MCMC has the following set of 13 jump parameters: the planet-to-star flux ratios in the Kepler and Spitzer bandpasses, the transit depth, the impact parameter $b$, the transit duration from first to fourth contact, the time of minimum light, the orbital period, $K^{\prime}=K \sqrt{1-e^{2}} P^{1 / 3}$ where $K$ is the radial velocity semi-amplitude, the two LD combinations $c_{1}$ and $c_{2}$, and the two parameters $\sqrt{e} \cos \omega$ and $\sqrt{e} \sin \omega$ (Anderson et al. 2011). A uniform prior distribution is assumed for all jump parameters. Baseline model coefficients are determined for each light curve with the SVD method (Press et al. 1992) at each step of the 
Table 3

System Parameters for Kepler-17

\begin{tabular}{|c|c|c|}
\hline Parameter & Value & Notes \\
\hline \multicolumn{3}{|l|}{ Object of interest } \\
\hline Kepler Input Catalog number (KIC) & 10619192 & \\
\hline Kepler Object of Interest number (KOI) & 203 & \\
\hline R.A. (J2000) & $19: 53: 34.86$ & \\
\hline Decl. (J2000) & $+47: 48: 54.0$ & \\
\hline Kepler magnitude & 14.14 & \\
\hline$J$ magnitude & 12.99 & \\
\hline$r$ magnitude & 14.08 & \\
\hline \multicolumn{3}{|l|}{ Transit and orbital parameters } \\
\hline Orbital period $P$ (days) & $1.4857108 \pm 0.0000002$ & A \\
\hline Transit Ephemeris $\left(\mathrm{BJD}_{\mathrm{utc}}\right)$ & $2455185.678035_{-0.000026}^{+0.000023}$ & A \\
\hline Scaled semimajor axis $a / R_{\star}$ & $5.48 \pm 0.02$ & A \\
\hline Scaled planet radius $R_{\mathrm{P}} / R_{\star}$ & $0.13031_{-0.00018}^{+0.00022}$ & A \\
\hline Impact parameter $b \equiv a \cos i / R_{\star}$ & $0.268_{-0.012}^{+0.014}$ & A \\
\hline Orbital inclination $i$ (deg) & $87.2 \pm 0.15$ & A \\
\hline Orbital semi-amplitude $K\left(\mathrm{~m} \mathrm{~s}^{-1}\right)$ & $419.5_{-156}^{+13.3}$ & $\mathrm{~A}, \mathrm{~B}$ \\
\hline$\sqrt{e} \cos (w)$ & $0.008_{-0.013}^{+0.0 .10}$ & $\mathrm{~A}, \mathrm{~B}$ \\
\hline$\sqrt{e} \sin (w)$ & $-0.084_{-0.033}^{+0.027}$ & $\mathrm{~A}, \mathrm{~B}$ \\
\hline Orbital eccentricity $e$ & $<0.011$ & $\mathrm{~A}, \mathrm{~B}$ \\
\hline Transit duration (days) & $0.09485_{-0.00007}^{+0.00007}$ & \\
\hline \multicolumn{3}{|c|}{ Observed stellar parameters (fixing the surface gravity) } \\
\hline Effective temperature $T_{\text {eff }}(\mathrm{K})$ & $5630 \pm 100$ & $\mathrm{C}$ \\
\hline Metallicity $[\mathrm{M} / \mathrm{H}]$ & $0.3 \pm 0.1$ & $\mathrm{C}$ \\
\hline Projected rotation $v \sin i\left(\mathrm{~km} \mathrm{~s}^{-1}\right)$ & $4.7 \pm 1.0$ & $\mathrm{C}$ \\
\hline Absolute systemic radial velocity $\gamma\left(\mathrm{km} \mathrm{s}^{-1}\right)$ & $-23.82 \pm 0.10$ & $\mathrm{C}$ \\
\hline Mean radial velocity $\left(\mathrm{km} \mathrm{s}^{-1}\right)$ & $+0.40 \pm 0.10$ & $\mathrm{~B}$ \\
\hline Quadratic limb-darkening coefficient $\left(u_{1}\right)$ & $0.405 \pm 0.007$ & A \\
\hline Quadratic limb-darkening coefficient $\left(u_{2}\right)$ & $0.262_{-0.015}^{+0.013}$ & A \\
\hline \multicolumn{3}{|l|}{ Derived stellar parameters } \\
\hline Mass $M_{\star}\left(M_{\odot}\right)$ & $1.06 \pm 0.07$ & C,D \\
\hline Radius $R_{\star}\left(R_{\odot}\right)$ & $1.02 \pm 0.03$ & C,D \\
\hline Surface gravity $\log g_{\star}(\mathrm{cgs})$ & $4.43 \pm 0.02$ & A \\
\hline Age (Gyr) & $3 \pm 1.6$ & $\mathrm{C}, \mathrm{D}$ \\
\hline \multicolumn{3}{|l|}{ Planetary parameters } \\
\hline Mass $M_{\mathrm{P}}\left(M_{\mathrm{J}}\right)$ & $2.45 \pm 0.11$ & $\mathrm{~A}, \mathrm{~B}, \mathrm{C}, \mathrm{D}$ \\
\hline Radius $R_{\mathrm{P}}\left(R_{\mathrm{J}}\right.$, equatorial $)$ & $1.31 \pm 0.02$ & $\mathrm{~A}, \mathrm{~B}, \mathrm{C}, \mathrm{D}$ \\
\hline Density $\rho_{\mathrm{P}}\left(\mathrm{g} \mathrm{cm}^{-3}\right)$ & $1.35 \pm 0.08$ & $\mathrm{~A}, \mathrm{~B}, \mathrm{C}, \mathrm{D}$ \\
\hline Surface gravity $\log g_{\mathrm{P}}(\mathrm{cgs})$ & $3.55 \pm 0.02$ & $\mathrm{~A}, \mathrm{~B}$ \\
\hline Orbital semimajor axis $a(\mathrm{AU})$ & $0.02591_{-0.00036}^{+0.00037}$ & $\mathrm{E}$ \\
\hline Equilibrium temperature $T_{\mathrm{eq}}(\mathrm{K})$ & $1570 \pm 200$ & $\mathrm{~F}$ \\
\hline Geometric albedo $A_{g}$ & $0.10 \pm 0.02$ & A \\
\hline
\end{tabular}

Notes. A: based on the photometry. B: based on the radial velocities. C: based on an analysis of the TRES spectrum. D: based on the Yale-Yonsei stellar evolution tracks. E: based on Newton's version of Kepler's third law and total mass. F: assumes Bond albedo $=0$ and complete redistribution.

MCMC. Correlated noise is accounted for following Winn et al. (2008) and Gillon et al. (2010) to ensure reliable error bars on the fitted parameters. For this purpose, we compute a scaling factor based on the standard deviation of the binned residuals for each light curve with different time bins. The error bars are then multiplied by this scaling factor. We obtained a mean scaling factor of 1.19 for all photometry. The results from the MCMC analysis are presented in Table 3.

\section{RESULTS AND DISCUSSION}

\subsection{Characteristics of the System Kepler-17}

Adopting the stellar mass and radius for Kepler-17 that we found in Section 4, we obtain a mass of $M_{\mathrm{P}}=2.45 \pm 0.11 M_{\mathrm{J}}$ and a radius of $R_{\mathrm{P}}=1.31 \pm 0.02 R_{\mathrm{J}}$ for the planet, which leads to a density of $\rho_{\mathrm{P}}=1.35 \pm 0.08 \mathrm{~g} \mathrm{~cm}^{-3}$ and a surface gravity $\log g_{\mathrm{P}}=3.55 \pm 0.02$ (cgs). We note that the surface gravity can be derived from the photometry and the velocimetry only (Southworth et al. 2007). The position of Kepler-17b on the mass-radius diagram is fairly common and it appears to be slightly inflated compared to models that include the effects of stellar irradiation (e.g., Latham et al. 2010).

The current upper limit on the orbital eccentricity $e$ from radial velocity measurements is consistent with zero for Kepler-17b. We also measure the mid-occultation timing offset from both Kepler and Spitzer observations. The determination of the timing of the secondary eclipse constrains the planet's orbital eccentricity. Our estimate for the best-fit timing offset translates to a constraint on $e$ and the argument of pericenter $\omega$. The timing is used to constrain the $e \cos (\omega)$ and we find that 

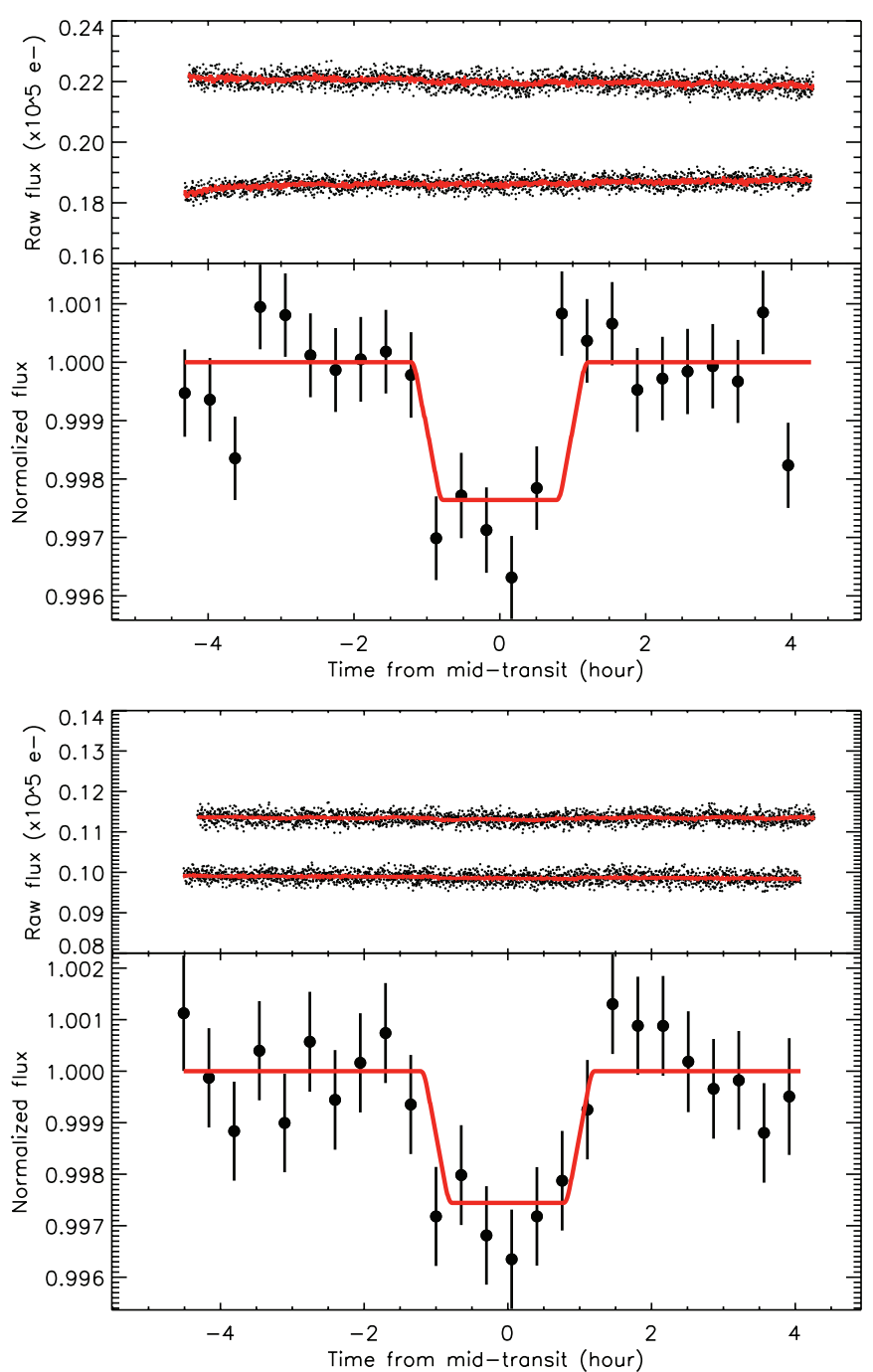

Figure 7. Spitzer occultation light curves of Kepler-17 observed in the IRAC bandpass at 3.6 (top) and $4.5 \mu \mathrm{m}$ (bottom). Two visits were obtained for each Spitzer bandpass. Top panels: raw and unbinned light curves. The red solid lines correspond to the best-fit models which include the time and position instrumental decorrelations as well as the model for the planetary occultations (see details in Section 5.2). Bottom panels: corrected and normalized occultation light curves with their best-fit models (in red). The data are binned in 25 minute intervals (50 data points per bin).

(A color version of this figure is available in the online journal.)

$e<0.011$ at the $1 \sigma$ level. This upper limit implies that the orbits of these objects are nearly circular unless the line of sight is aligned with the planet's major orbital axis, i.e., the argument of periapse $\omega$ is close to $90^{\circ}$ or $270^{\circ}$.

We fit a linear function of transit epoch and find $T_{c}(0)=2455185.678035_{-0.000026}^{+0.000023}\left(\mathrm{BJD}_{\text {utc }}\right)$ with a period $P=$ $1.4857108 \pm 0.0000002$ days. We also fit each mid-transit time individually and compare each one to the expected linear ephemeris to obtain a observed-minus-computed $(O-C)$ time series. The $O-C$ times show a scatter that is most probably caused by stellar spot-induced shifts, since the star is active (see Section 7.2). Formally, the individual fits are consistent with the linear model, therefore we do not consider this to be a significant detection of timing anomalies. Since there is no clear evidence for transit timing variations (TTVs; Agol et al. 2005; Holman \& Murray 2005), we use the timing data to place upper limits on the mass of a hypothetical second planet that would perturb the orbit of the transiting planet using the procedure

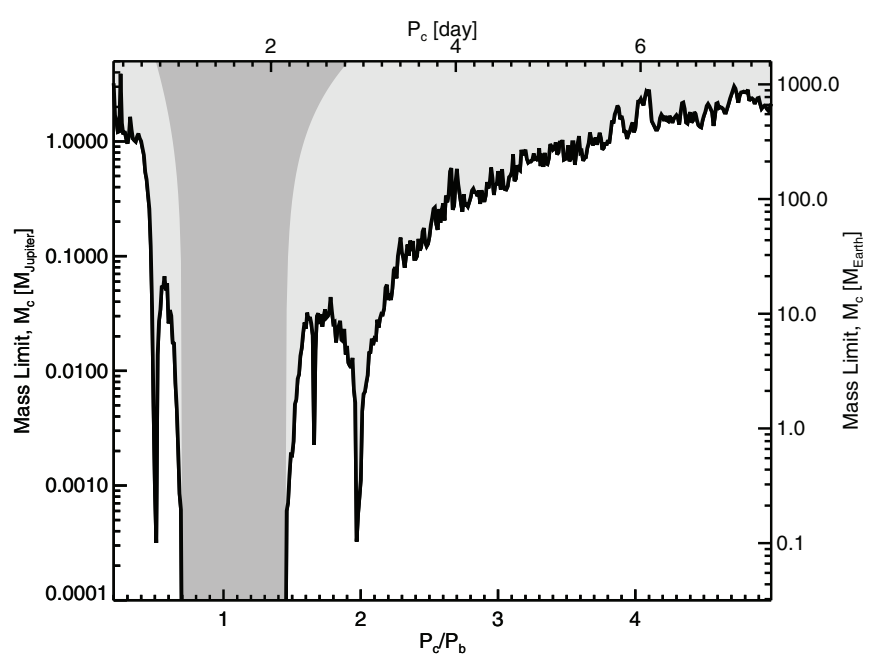

Figure 8. Upper mass limits for a hypothetical perturber as a function of perturber period normalized to the period of Kepler-17b, $P_{c} / P_{b}$. The dark gray zone corresponds to the region of dynamical instability, the white zone corresponds to the region where the presence of a companion with a minimum mass would be permitted by the current limits on TTVs.

described in Carter et al. (2011). Figure 8 shows the constraints on the perturber mass as a function of period ratio, as determined from this analysis. The mass constraints on the perturber are more restrictive near the mean-motion resonances and most restrictive at the low-order resonances, particularly for the interior and exterior 2:1 resonances. For example, a perturber at the interior 2:1 resonance having a mass near that of Mars would have induced TTVs detectable in the present data.

\subsection{Stellar Variability of Kepler-17}

The Kepler photometry exhibits a quasi periodic flux modulation of about $3 \%$ with a period of 11.9 days (Figure 1). This period corresponds to eight times the planet's orbital period (Figure 9). The stellar variability can be quantified from a Lomb-Scargle periodogram that reveals two peaks at 5.95 and 11.9 days (see Figure 10). The position of the peak on the periodogram and its width provide a measurement of the stellar rotation period and its error, respectively. We measured a stellar rotation period of $11.9 \pm 1.1$ days. The presence of active stellar spots localized on opposite hemispheres can best explain the two peaks seen in the periodogram. We find that the ratio between the planet's orbital period and the stellar rotation period is $8.0 \pm 0.7$ days. We note that this intriguing integer ratio of 8 could potentially reveal the signature of stellar-planet interactions.

\subsubsection{Using Occulted Starspots to Infer the Orbital Obliquity}

The light curve of Kepler-17 shows substantial deformation of the planet's transit profiles which we interpret as occultations of dark starspots (Figure 2). We co-added the transit light curves that occurred at epochs modulo the stellar rotation period (modulo 8 planetary orbital periods). We present the resulting eight transit light curves in Figure 11; each contains about 22 individual transit light curves. We identified that the planet crosses spots of identical shapes every eight transits, producing a stroboscopic effect. Therefore, we conclude that starspots return to the same position on the transit chord after eight orbits or 11.89 days (one stellar rotation period). Because of this stroboscopic effect, the scatter measured during the spotcrossing phase of each of these light curve residuals is similar 


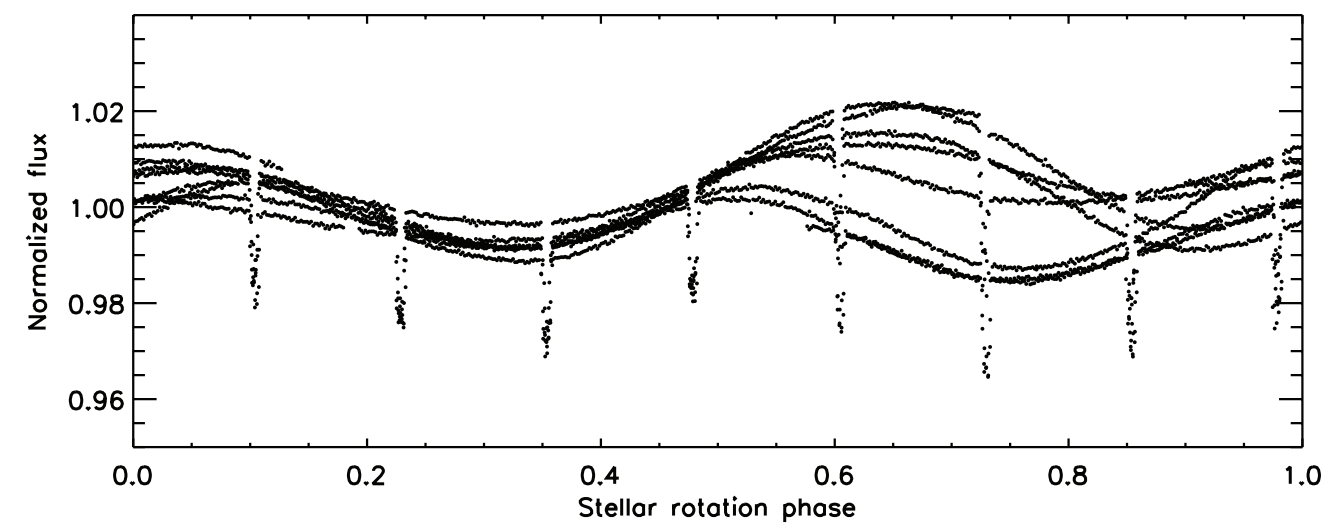

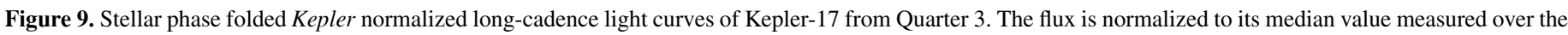

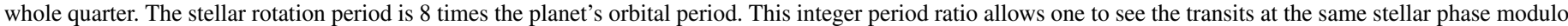
8. The stellar variability exhibits $3 \%$ flux variation with a period of approximately 11.9 days.

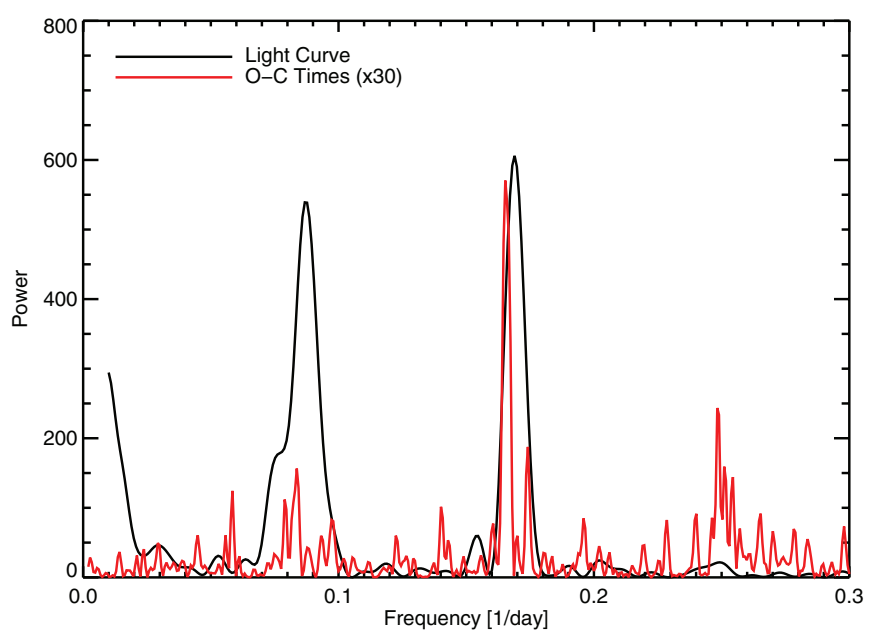

Figure 10. Lomb-Scargle periodograms of the Kepler photometry (in black) and of the observed-minus-computed $(O-C)$ mid-transit times (in red) magnified by 30 for comparison purposes. The periodogram from the photometry shows two main peaks at 5.95 and 11.9 days while the $O-C$ periodogram exhibits a dominant peak at 6 days.

(A color version of this figure is available in the online journal.)

to the scatter of the residuals measured outside the transit. This further supports the assertion that the same spots are crossed by the planet every eight transits, at identical orbital phases, because the residuals measured from a transit light curve obtained from a planet occulting a random distribution of spots are expected to be larger during the transit event compared to the out-of-transit monitoring. This can be explained only if spots are coming back to similar longitudes and latitudes after one stellar rotation period. Interestingly, occulted stellar spots can place constraints on the spin-orbit alignment (e.g., Deming et al. 2011; Nutzman et al. 2011; Sanchis-Ojeda et al. 2011b; Sanchis-Ojeda \& Winn 2011a).

We identified five spots (A, B, C, D, and E) by their anomaly in the residuals and we marked their initial orbital phase positions as seen in Figure 11. We then computed the expected phase position of each of these five anomalies on the eight following light curve residuals assuming that occulted starspots should have moved by $360 / 8=45^{\circ}$ in longitude toward the egress (for a prograde and aligned orbit) for every planetary transit. We find that anomalies are always present at all the expected phases. As the star rotates, the spot surfaces projected on the stellar sphere change; therefore the shapes and amplitudes of these anomalies change between consecutive transits while the same spots are crossed over time. Since a spot surface appears larger at the center of the star compared to the limb, the anomalies due to spots should exhibit larger amplitudes when they are crossed close to the mid-transit time. This is consistent with what is observed in Kepler-17. For example, anomaly " $B$ " in Figure 11 is observed in four consecutive transits. The overall shape of this anomaly is conserved transit after transit and its amplitude increases before and decreases after the mid-transit. We conclude that the planet crosses the same spots, transit after transit, at longitudinal positions that differ by $45^{\circ}$.

These findings imply that the projected spin-orbit angle, $\lambda$, is very close to 0 for this system. Because occulted spots can be identified transit after transit (Figure 12), with a change in phase expected from the stellar rotation period, we conclude that the stellar inclination angle is near $90^{\circ}$. This is further supported by the measured $v \sin i=4.7 \pm 1.0 \mathrm{~km} \mathrm{~s}^{-1}$, which is in good agreement with its expected value of $2 \pi R_{\star} / P_{\text {rot }}=4.3 \mathrm{~km} \mathrm{~s}^{-1}$ assuming an inclination angle of $90^{\circ}$. This implies that the true obliquity, i.e., the angle between the stellar rotational axis and a line perpendicular to planet's orbital plane, $\Psi$, must be close to 0 . Finally, we conclude that the planet orbit is prograde since the occulted starspots progress from the transit ingress to the egress limbs.

Assuming that the uncertainty on latitude of a spot corresponds to the planet size, we derive an uncertainty for $\Psi$ of $\pm \arctan \left(R_{\mathrm{P}} / R_{\star}\right)= \pm 10^{\circ}$. We note that the spot size could be larger than the planet size, but this changes the results only modestly. Assuming a starspot twice the projected size of the planet on the stellar photosphere provides a more conservative uncertainty of $\pm 15^{\circ}$ for $\Psi$.

Winn et al. (2010) show that stars with transiting planets for which the stellar obliquity is large are preferentially hot $\left(T_{\text {eff }}>6250 \mathrm{~K}\right)$. The low obliquity of Kepler- $17 \mathrm{~b}$ fits this pattern since the stellar temperature is lower than $6250 \mathrm{~K}$.

\subsubsection{Starspots Lifetime}

To help visualize the spot crossings during transit, we subtract the best-fit model from each individual transit and investigate the residuals. We slide a box of duration twice the ingress time across the residuals in each transit epoch, recording the scatter for a given box mid-time (relative to mid-transit) and a given epoch. We then produce an image of this scatter at orbital phase as a function of the epoch number (see Figure 12). We used an interpolation (with IDL's TRIGRID) with an output sampling 

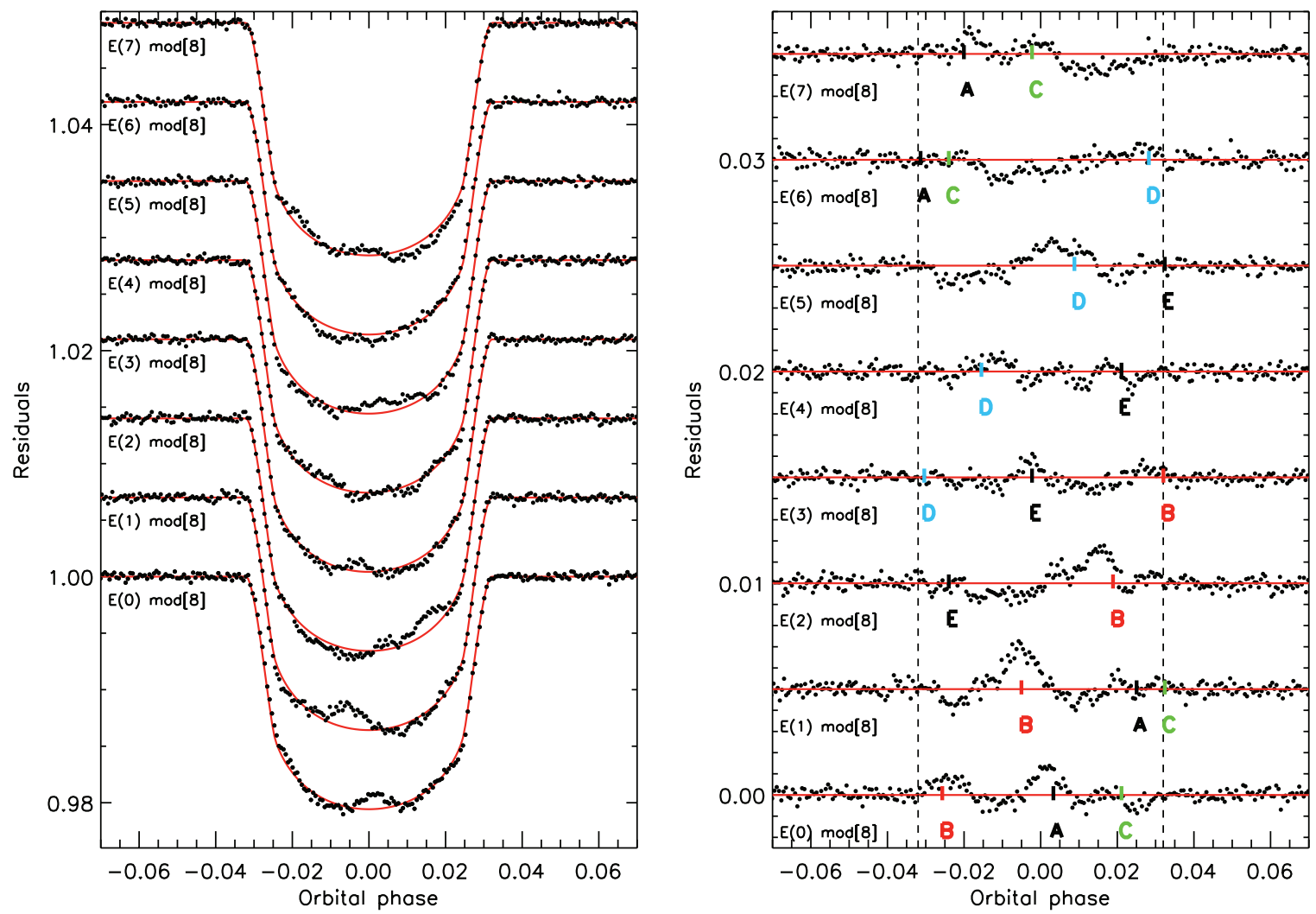

Figure 11. Left: sequence of combined and binned transit light curves, with the best-fit model presented in Figure 2 and overplotted in red. Each co-added transit corresponds to the combination of 22 individual transits that occurred at epochs modulo eight planetary orbital periods. The light curves are binned by $100 \mathrm{~s}$ and they are shifted vertically for display purposes. Each combination of individual transits allows us to increase the $\mathrm{S} / \mathrm{N}$ and to demonstrate that the same spots are occulted during several consecutives transits and epochs. The overall combination of these eight transit light curves gives the final curves presented in Figure 2. Occulted stellar spots are revealed in the combined curves since the stellar rotation period is eight times the planet's orbital period. The same spots are crossed every eight transits at a similar orbital phase. Right: residuals of the best-fit model subtracted from each individual combined light curve modulo 8 . The vertical dashed lines correspond to the beginning and to the end of the transits. Five occulted stellar spots are indicated on the residuals (A, B, C, D, and E) as they appear transit after transit at phase positions expected from the stellar rotation period. This implies that the projected spin-orbit angle, $\lambda$, is very close to 0 for this system. The combination of the residuals of the eight transit light curves is similar to the total residuals plotted in Figure 2 and exhibits a symmetrical structure.

(A color version of this figure is available in the online journal.)

of 600 samples in the epochal direction and 300 samples in the transit phase direction. This image reveals individual spots that we define as either "hot" or "cold" regions, depending on whether the individual slide box residuals are below or above the transit light curve model. The repeating vertical structure is interpreted as spots marching across the transit chord such as seen in the previous section. Each vertical profile is slanted slightly from left to right indicating that the spots progress from the ingress limb to the egress limb. Some spots make their way around the star and reappear again during several stellar rotation periods. For example, the collection of "cold" spots in the image starting around Epoch 110 and ending around Epoch 170 seems to be related to the same spot. We conclude from the nearly continuous monitoring of Kepler-17 that the occulted starspots are present on the same stellar chord for at least 100 days, somewhat comparable to the lifetime of sunspots.

As Kepler continues to monitor transits of hot Jupiters in front of active stars, it will help to better understand the stellar cycles. If the Kepler mission is extended, the long term photometry will enable it to produce starspot maps and learn more about spot mean lifetimes and photospheric differential rotations. In the case of Kepler-17, we may be able to measure the complete activity cycle for this star and to compare it to another well-know G-dwarf: the Sun.

\subsubsection{Impact of Stellar Variability of Kepler-17 on the System Parameters}

When the planet transits in front of stellar spots, its transit shape deviates from the averaged phase-folded light curve. The effect of occulted stellar spots on the shape of the transit light curve is observed in the residuals from the best-fit transit model of the phase-folded light curve (see Figure 2). Since the stellar activity influences the transit light curve profiles, the planetary parameters we derive from these profiles are likely to be affected. This is a well-known problem for planets transiting in front of variable stars (e.g., Czesla et al. 2009; Désert et al. 2011a). Importantly for the present study, the variability affects the stellar density that we assume a fixed value for our determination of the stellar parameters (see Section 4). Czesla et al. (2009) propose to fit the lower envelope of the transit light curve to recover more realistic transit parameters. This assumes that dark stellar structures dominate over bright faculae. In the case of Kepler-17b, we cannot exclude the possibility that every transit is affected by dark or bright stellar regions so that a priori no individual transit light curve can be used as representative of an unaffected profile. Furthermore, because of the stroboscopic effect described above, the phase-folded transit light curve possesses combined pattern distortions that prevents the use of its lower envelope to derive more accurate parameters. 


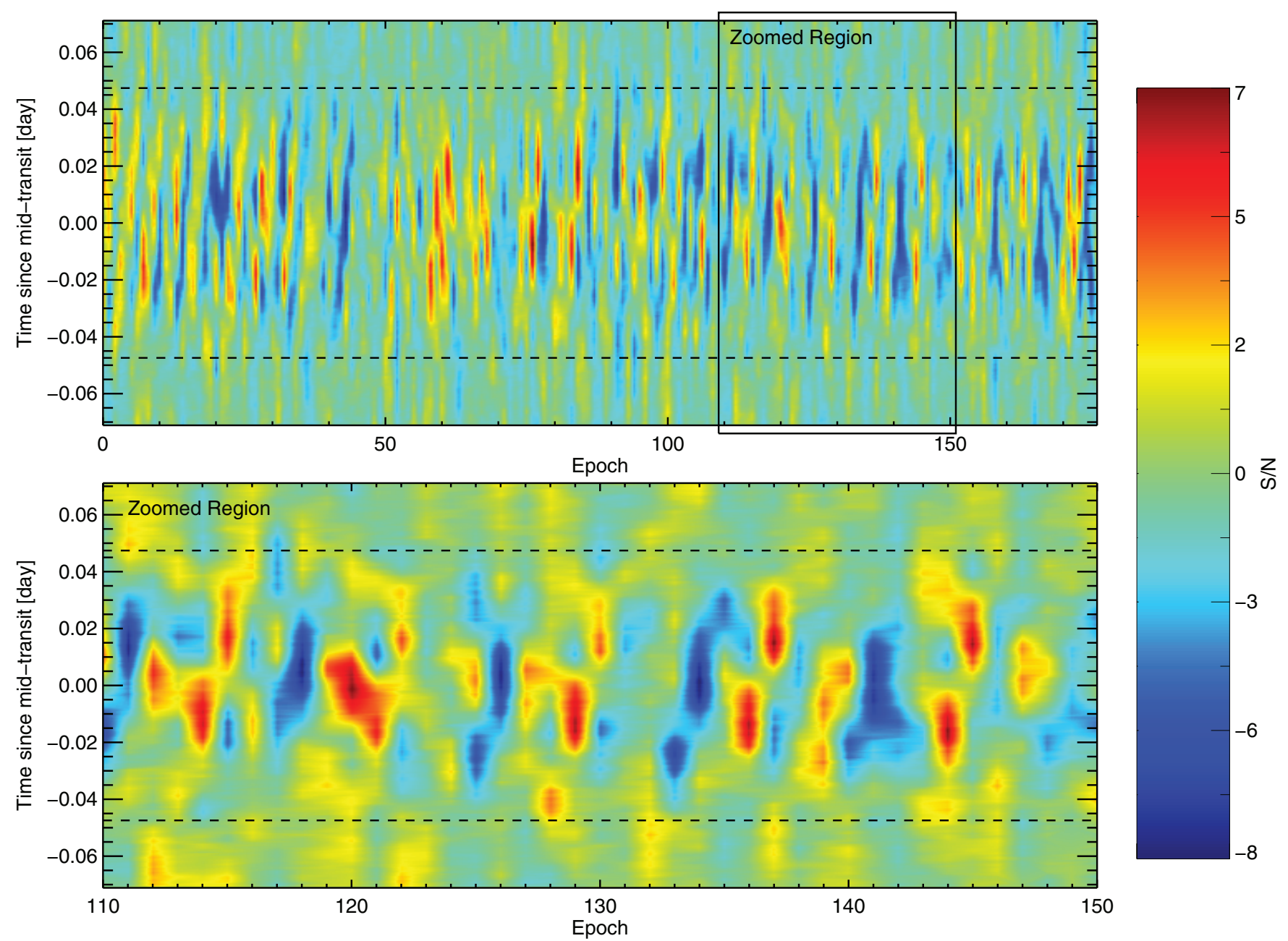

Figure 12. Lifetime of stellar spots occulted during transits. The top panel corresponds to the complete map constructed from Quarter 1 to 6 data. The bottom panel is a zoom of the top panel. This time-map image corresponds to the scatter $(\mathrm{S} / \mathrm{N})$ measured in a sliding box of duration twice the ingress time across the residuals in each transit epoch, relative to mid-transit ( $Y$-axis) as a function of the epoch ( $X$-axis). The regions in blue correspond to times when the sections of individual transit light curves are deeper than the averaged transit light curve, whereas the regions in red correspond to less deep sections attributed to cold stellar spots. For display purposes, we used an interpolation with an output sampling of 600 samples in the epochal direction and 300 samples in the transit phase direction.

(A color version of this figure is available in the online journal.)

We note that the combination of the residuals for the eight transit light curves presented in Figure 11 is very similar to the total residuals plotted in Figure 2 and exhibits a symmetrical structure. This symmetrical pattern has a shape consistent with what would be expected from an oblate planet but with an amplitude ten to a hundred times larger than expected for a Jupiter size planet (Seager \& Hui 2002; Barnes \& Fortney 2003; Carter \& Winn 2010). This pattern represents a remarkable coincidence, in that it seems quite symmetric but it is only obtained when all eight time series are combined, and does not appear in the residuals for any single time series.

\subsection{Atmospheric Constraints for Kepler-17b}

The occultation depths measured in each bandpass are combined and turned into an emergent spectrum for the planet. The observed flux of the planet in each bandpass corresponds to the sum of the reflected light and the thermally emitted light. The combined occultation observed in the Kepler bandpass provides a measure of the planet's geometric albedo. The occultation depth of $58 \pm 8 \mathrm{ppm}$ corresponds to $A_{g}=0.10 \pm 0.02$. Assuming a Lambertian criterion $A_{\mathrm{B}} \leqslant 1.5 \times A_{\mathrm{g}}$, and assuming that the geometric albedo measured in the Kepler bandpass is the same at every wavelength, we infer an upper limit to the Bond albedo of $A_{\mathrm{B}} \leqslant 0.18$ at the $1 \sigma$ level. This result is conservative in the sense that the true albedo may be even lower, if some of the occultation signal we have measured in the Kepler bandpass is due to thermal emission rather than reflected light. This is expected for the temperature regime of hot-Jupiters. Therefore, the Bond albedo could be well below 0.18.

We estimate the thermal component of the planet's emission in the two Spitzer bandpasses from the occultation depths measured at 3.6 and $4.5 \mu \mathrm{m}$ (see Table 2). We assume that the planetary emission is well reproduced by a blackbody spectrum and translate the measured depth of the secondary eclipse into brightness temperatures. We use the PHOENIX atmospheric code (Hauschildt et al. 1999) to produce theoretical stellar models for the star Kepler-17. Taking the Spitzer spectral response function into account, the ratio of areas of the star and the planet and the stellar spectra, we derive the brightness temperatures that best fit the observed eclipse depths measured in the two IRAC bandpasses. The brightness temperature calculated this way result in $T_{3.6 \mu \mathrm{m}}=1880 \pm 110 \mathrm{~K}$ and $T_{4.5} \mu \mathrm{m}=1770 \pm 150 \mathrm{~K}$.

We compare our data to the hot-Jupiter atmospheric model described in Fortney et al. (2008) which has been used for a 


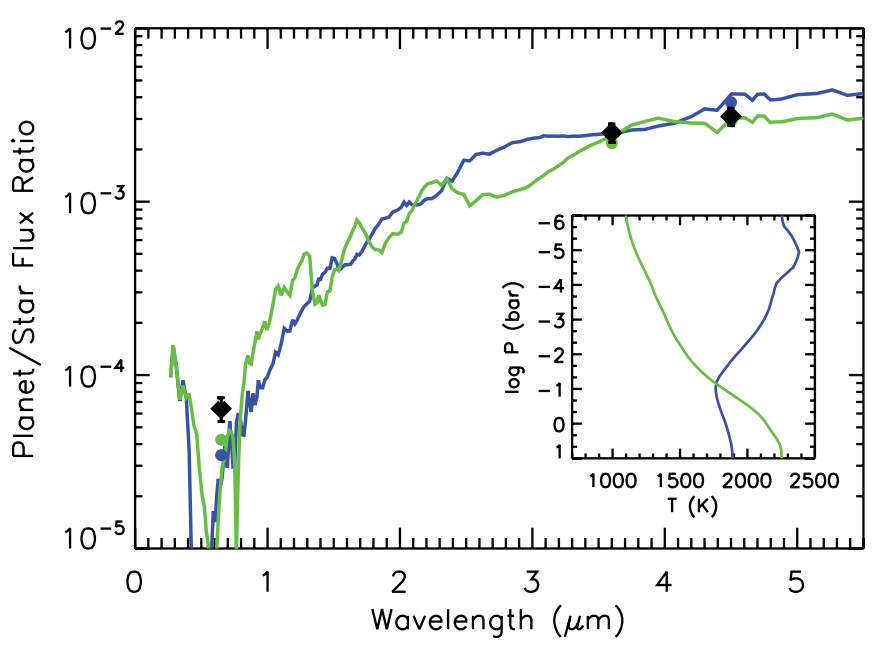

Figure 13. Day side planet-to-star flux ratios as function of the wavelength for two atmospheric models (Fortney et al. 2008). The black filled diamonds and their error bars correspond to the Kepler and Spitzer observations. The data set is best fitted with models assuming full redistribution of the energy to the planetary day side. The green model represents a non-inverted atmosphere (no $\mathrm{TiO}$ ). The blue model represents an inverted atmosphere (with $\mathrm{TiO}$ ). The target is slightly better fitted by the non-inverted model.

(A color version of this figure is available in the online journal.)

variety of close-in planets (Fortney et al. 2005, 2006). We aim at broadly distinguishing between different classes of model atmospheres, given that there are no prior constraints on the basic composition and structure. The atmospheric spectrum calculations are performed for 1D atmospheric pressure-temperature $(P-T)$ profiles and use the equilibrium chemical abundances, at solar metallicity, described in Lodders (2002) and Lodders \& Fegley (2006). This is a self-consistent treatment of radiative transfer and chemical equilibrium of neutral species. The opacity database is described in Freedman et al. (2008). The models are calculated for various values of the dayside-to-nightside energy redistribution parameter $(f)$ and allow for the presence of $\mathrm{TiO}$ at high altitude, which may play the role of an absorber and which likely leads to an inversion of the $P-T$ profile (Figure 13).

We compare the data to model predictions and select models with the best reduced $\chi^{2}$. We note that this is not a fit involving adjustable parameters. All models shown assume redistribution of absorbed flux over the day side only, favoring low values for $f$. Since our observations are only weakly constraining, the 3.6 and $4.5 \mu \mathrm{m}$ depth ratios are best fitted by a model without a temperature inversion, although the model with a temperature inversion is a reasonable fit as well. In the model with no inversion the optical opacity sources are neutral atomic $\mathrm{Na}$ and $\mathrm{K}$, while in the inverted model the optical opacity is dominated by gaseous $\mathrm{TiO}$ and $\mathrm{VO}$ molecules. The relatively large occultation depth in the Kepler bandpass is not matched by these simple models. This may indicate that silicate clouds (as is seen in L-type brown dwarfs, but not modeled here) may lead to a higher amount of scattered flux from the planet. It may also indicate that optical opacity sources are overestimated, such that more planetary thermal flux is being seen from deeper layers. The growing sample size of Kepler occultation detections may show trends with planetary $T_{\text {eff }}$ that could help to clarify this issue.

Hartman (2010) shows that there is a correlation between the surface gravity of hot Jupiters and the activity levels of the host stars, such that high surface gravity planets tend to be found around high-activity stars. The position of Kepler-17b on such a diagram is consistent with this trend. Knutson et al. (2010) show that there could also be a correlation between the host star activity level and the thermal inversion of the planetary atmosphere. In this picture, the strong XEUV irradiation from the active stellar host of a hot-Jupiter depletes the atmosphere of chemical species responsible for producing inversions. The $\log R_{\mathrm{HK}}^{\prime}$ of Kepler-17 is consistent with the case of no thermal inversion in the framework developed by Knutson et al. (2010). We also evaluated the empirical index defined by Knutson et al. (2010), which could be correlated with the presence of a thermal inversion. Using the same definition, we find indices of $0.026 \%$ above and $0.034 \%$ below the predicted blackbody fluxes in the 3.6 and $4.5 \mu \mathrm{m}$ bands. These indices suggest that the atmosphere of Kepler-17b is consistent with a non-inverted profile, which is in agreement with the results of our present study.

This work is based on observations made with Kepler, which was competitively selected as the tenth Discovery mission. Funding for this mission is provided by NASA's Science Mission Directorate. The authors thank the many people who generously gave so much their time to make this Mission a success.

This work is also based on observations made with the Spitzer Space Telescope, which is operated by the Jet Propulsion Laboratory, California Institute of Technology under a contract with NASA. Support for this work was provided by NASA through an award issued by JPL/Caltech.

Some of the data presented herein were obtained at the W. M. Keck Observatory, which is operated as a scientific partnership among the California Institute of Technology, the University of California and the National Aeronautics and Space Administration. The Observatory was made possible by the generous financial support of the W. M. Keck Foundation.

This work is also based on observations obtained with the Hobby-Eberly Telescope (HET), which is a joint project of the University of Texas at Austin, the Pennsylvania State University, Stanford University, Ludwig-Maximilians-Universitat Munchen, and Georg-August-Universitat Gottingen. The HET is named in honor of its principal benefactors, William P. Hobby and Robert E. Eberly.

We thank the Spitzer staff at IPAC and in particular Nancy Silbermann for scheduling the Spitzer observations of this program.

M.G. is an FNRS Research Associate.

\section{REFERENCES}

Agol, E., Steffen, J., Sari, R., \& Clarkson, W. 2005, MNRAS, 359, 567

Alonso, R., Deeg, H. J., Kabath, P., \& Rabus, M. 2010, AJ, 139, 1481

Alonso, R., Guillot, T., Mazeh, T., et al. 2009, A\&A, 501, L23

Anderson, D. R., Collier, C. A., Hellier, C., et al. 2011, ApJ, 726, L19

Barman, T. S., Hauschildt, P. H., \& Allard, F. 2005, ApJ, 632, 1132

Barnes, J. W., \& Fortney, J. J. 2003, ApJ, 588, 545

Batalha, N. M., Borucki, W. J., Koch, D. G., et al. 2010, ApJ, 713, L109

Borucki, W. J., Koch, D., Basri, G., et al. 2010, Science, 327, 977

Borucki, W. J., Koch, D. G., Basri, G., et al. 2011, ApJ, 736, 19

Borucki, W. J., Koch, D., Jenkins, J., et al. 2009, Science, 325, 709

Burrows, A., Hubeny, I., \& Sudarsky, D. 2005, ApJ, 625, L135

Burrows, A., Hubeny, I., Budaj, J., Knutson, H. A., \& Charbonneau, D. 2007, ApJ, 668, L171

Burrows, A., Ibgui, L., \& Hubeny, I. 2008, ApJ, 682, 1277

Brown, T. M., Latham, D. W., Everett, M. E., \& Esquerdo, G. A. 2011, AJ, 142 , 112

Bryson, S. T., Tenenbaum, P., Jenkins, J. M., et al. 2010, ApJ, 713, L97

Buchhave, L. A., Bakos, G. Á., Hartman, J. D., et al. 2010, ApJ, 720, 1118

Caldwell, D. A., Kolodziejczak, J. J., Van Cleve, J. E., et al. 2010, ApJ, 713, L92

Carter, J. A., \& Winn, J. N. 2010, ApJ, 709, 1219 
Carter, J. A., Winn, J. N., Holman, M. J., et al. 2011, ApJ, 730, 82

Charbonneau, D., Allen, L. E., Megeath, S. T., et al. 2005, ApJ, 626, 523

Czesla, S., Huber, K. F., Wolter, U., Schröter, S., \& Schmitt, J. H. M. M. 2009, A\&A, 505, 1277

Demarque, P., Woo, J.-H., Kim, Y.-C., \& Yi, S. K. 2004, ApJS, 155, 667

Deming, D., Seager, S., \& Richardson, L. J. 2005, Nature, 434, 740

Deming, D., et al. 2011, ApJ, in press (arXiv:1107.2977)

Désert, J.-M., Lecavelier des Etangs, A., Hébrard, G., et al. 2009, ApJ, 699, 478

Désert, J.-M., Sing, D., Vidal-Madjar, A., et al. 2011a, A\&A, 526, A12

Désert, J.-M., et al. 2011b, ApJS, 197, 11

Endl, M., MacQueen, P. J., Cochran, W. D., et al. 2011, ApJS, 197, 13

Fazio, G. G., Hora, J. L., Allen, L. E., et al. 2004, ApJS, 154, 10

Fortney, J. J., Cooper, C. S., Showman, A. P., Marley, M. S., \& Freedman, R. S. 2006, ApJ, 652, 746

Fortney, J. J., Lodders, K., Marley, M. S., \& Freedman, R. S. 2008, ApJ, 678, 1419

Fortney, J. J., Marley, M. S., Lodders, K., Saumon, D., \& Freedman, R. 2005, ApJ, 627, L69

Freedman, R. S., Marley, M. S., \& Lodders, K. 2008, ApJS, 174, 504

Fürész, G. 2008, PhD thesis, Univ. Szeged, Hungary

Gautier, T. N., III, Batalha, N. M., Borucki, W. J., et al. 2010, arXiv:1001.0352

Gilliland, R. L., Jenkins, J. M., Borucki, W. J., et al. 2010, ApJ, 713, L160

Gillon, M., Demory, B.-O., Triaud, A. H. M. J., et al. 2009, A\&A, 506, 359

Gillon, M., Lanotte, A. A., Barman, T., et al. 2010, A\&A, 511, A3

Hartman, J. D. 2010, ApJ, 717, L138

Hauschildt, P. H., Allard, F., \& Baron, E. 1999, ApJ, 512, 377

Holman, M. J., \& Murray, N. W. 2005, Science, 307, 1288

Holman, M. J., Winn, J. N., Latham, D. W., et al. 2006, ApJ, 652, 1715

Isaacson, H., \& Fischer, D. 2010, ApJ, 725, 875

Jefferys, W. H., Fitzpatrick, M. J., \& McArthur, B. E. 1988, Celestial Mechanics, 41, 39

Jenkins, J. M., Caldwell, D. A., Chandrasekaran, H., et al. 2010a, ApJ, 713, L120

Jenkins, J. M., Caldwell, D. A., Chandrasekaran, H., et al. 2010b, ApJ, 713, L87

Knutson, H. A., Charbonneau, D., Allen, L. E., et al. 2008, ApJ, 673, 526

Knutson, H. A., Howard, A. W., \& Isaacson, H. 2010, ApJ, 720, 1569
Koch, D. G., Borucki, W. J., Basri, G., et al. 2010a, ApJ, 713, L79

Koch, D. G., Borucki, W. J., Rowe, J. F., et al. 2010b, ApJ, 713, L131

Kurucz, R. L. 1979, ApJS, 40, 1

Latham, D. W., Borucki, W. J., Koch, D. G., et al. 2010, ApJ, 713, L140

Lodders, K. 2002, ApJ, 577, 974

Lodders, K., \& Fegley, B., Jr. 2006, in Astrophysics Update 2, ed. John W. Mason (Heidelberg: Springer), 1

Nidever, D. L., Marcy, G. W., Butler, R. P., Fischer, D. A., \& Vogt, S. S 2002, ApJS, 141, 503

Nutzman, P. A., Fabrycky, D. C., \& Fortney, J. J. 2011, ApJ, submitted (arXiv:1107.2106)

Press, W. H., Teukolsky, S. A., Vetterling, W. T., \& Flannery, B. P. 1992, in (2nd ed.; Cambridge: Cambridge Univ. Press)

Queloz, D., Henry, G. W., Sivan, J. P., et al. 2001, A\&A, 379, 279

Ramsey, L. W., Adams, M. T., Barnes, T. G., et al. 1998, Proc. SPIE, 3352, 34

Reach, W. T., et al. 2006, IRAC Data Handbook, version 3.0

Rowe, J. F., Matthews, J. M., Seager, S., et al. 2006, ApJ, 646, 1241

Rowe, J. F., Matthews, J. M., Seager, S., et al. 2008, ApJ, 689, 1345

Sanchis-Ojeda, R., \& Winn, J. N. 2011a, ApJ, in press (arXiv:1107.2920)

Sanchis-Ojeda, R., Winn, J. N., Holman, M. J., et al. 2011b, ApJ, 733, 127

Seager, S., \& Hui, L. 2002, ApJ, 574, 1004

Seager, S., \& Mallén-Ornelas, G. 2003, ApJ, 585, 1038

Seager, S., Whitney, B. A., \& Sasselov, D. D. 2000, ApJ, 540, 504

Snellen, I. A. G., de Mooij, E. J. W., \& Albrecht, S. 2009, Nature, 459, 543

Southworth, J. 2011, A\&A, submitted (arXiv:1107.1235)

Southworth, J., Wheatley, P. J., \& Sams, G. 2007, MNRAS, 379, L11

Sozzetti, A., Torres, G., Charbonneau, D., et al. 2007, ApJ, 664, 1190

Spiegel, D. S., \& Burrows, A. 2010, ApJ, 722, 871

Sudarsky, D., Burrows, A., \& Hubeny, I. 2003, ApJ, 588, 1121

Sudarsky, D., Burrows, A., \& Pinto, P. 2000, ApJ, 538, 885

Torres, G., Winn, J. N., \& Holman, M. J. 2008, ApJ, 677, 1324

Tull, R. G. 1998, Proc. SPIE, 3355, 387

Welsh, W. F., Orosz, J. A., Seager, S., et al. 2010, ApJ, 713, L145

Werner, M. W., Roellig, T. L., Low, F. J., et al. 2004, ApJS, 154, 1

Winn, J. N., Fabrycky, D., Albrecht, S., \& Johnson, J. A. 2010, ApJ, 718, L145

Winn, J. N., Holman, M. J., Torres, G., et al. 2008, ApJ, 683, 1076

Yi, S., Demarque, P., Kim, Y.-C., et al. 2001, ApJS, 136, 417 